\title{
Linx
}

LinX Revue des linguistes de l'université Paris X Nanterre

$73 \mid 2016$

Énonciation et marques d'oralité dans l'évolution du français

\section{L'évolution des marqueurs de type si ferai je, non ferai (XVIe-XVIIIe siècle)}

\section{Marta Saiz-Sánchez}

\author{
(2) OpenEdition \\ Journals \\ Édition électronique \\ URL : http://journals.openedition.org/linx/1636 \\ DOI : 10.4000/linx.1636 \\ ISSN : 2118-9692 \\ Éditeur \\ Presses universitaires de Paris Nanterre \\ Édition imprimée \\ Date de publication : 1 septembre 2016 \\ Pagination : 65-84 \\ ISSN : 0246-8743 \\ Référence électronique \\ Marta Saiz-Sánchez, «L'évolution des marqueurs de type si ferai je, non ferai (XVIe-XVIIIe siècle) », Linx \\ [En ligne], 73 | 2016, mis en ligne le 01 mars 2017, consulté le 19 avril 2019. URL : http:// \\ journals.openedition.org/linx/1636; DOI : 10.4000/linx.1636
}




\title{
L'évolution des marqueurs de type si ferai je, non ferai $\left(\mathrm{XVI}^{\mathrm{e}}-\mathrm{XVIII}{ }^{\mathrm{e}}\right.$ siècle $)$
}

\begin{abstract}
Résumé $^{1}$
Les expressions de type si/non + verbe substitut (+ sujet pronominal) du français médiéval sont des marqueurs du discours qui apparaissent dans le dialogue ou le monologue pour marquer l'accord et le désaccord avec ce qui a été dit précédemment. Ces structures présentent un fonctionnement argumentatif que oïl et nennil n'ont pas. À partir du français préclassique (1550), ces structures marquent uniquement le désaccord. Dans cet article nous traçons l'évolution des marqueurs du type si ferai je, non est il, si a, non fait, etc. en français préclassique et classique (du milieu du XVI à la fin du XVIII ${ }^{e}$ siècle). Le marqueur figé si fait, qui dès le français préclassique peut apparaître réduit au si de contradiction du français moderne, et le marqueur rédupliqué non, non héritent des valeurs sémantico-pragmatiques de la structure médiévale qui s'était désintégrée. Le théâtre classique connaitra les structures de type oh/ah/eh ! que + si/non qu'annonçaient les premières variations de si ferai je, non fait, etc. à la fin du français médiéval.
\end{abstract}

Abstract

The expressions of the si/non + substitute verb (+ pronominal subject) type in Medieval French are discourse markers which appear in a dialogue or a monologue to mark agreement and disagreement with something which was previously said. These structures present an argumentative use that oill and nennil do not have. From Preclassical French (1550) onwards, these structures exclusively mark disagreement. In this paper we describe the evolution of the markers of the type si ferai je, non est il, si a, non fait, etc. in Preclassical and Classical French (from the middle of the $16^{\text {th }}$ century until the end of the $18^{\text {th }}$ ). The marker si fait, which is fossilized, can be reduced from Preclassical French onwards to the contradiction particle si of Modern French. Si fait and the reduplicated marker non, non take over the semantic and pragmatic values of the medieval structure which had been disintegrated. Classical drama will use the structures of the oh/ah/eh ! que + si/non type that the first variations of si ferai je, non fait, etc. announced at the end of Medieval French.

\section{INTRODUCTION}

En français médiéval deux systèmes de marqueurs du discours permettent aux locuteurs de signaler qu'ils sont d'accord ou en désaccord avec un discours antérieur. D'un côté il y a ce que l'on appelle les pro-phrases (ou mots-phrase) ${ }^{2}$ oïl et nennil (oui et non), formées sur les particules latines hoc et ne suivies du pronom personnel il. D'un autre côté il y a les expressions de type si ferai je, non est il, si a, non fait, construites avec les adverbes si et non suivis d'un verbe substitut. Ces deux systèmes de marqueurs sont toujours réactifs, c'est-à-dire que, dans un dialogue, ils enchaînent sur ce que l'allocutaire a dit dans le tour de parole précédent. Dans un monologue, ces

\footnotetext{
${ }^{1}$ Cette contribution s'inscrit dans le cadre du Projet de recherche FFI201341355-P « Marqueurs pragmatiques et oralité en linguistique historique du français » du Ministerio de Economía y Competitividad, Espagne (Plan Estatal $\mathrm{I}+\mathrm{D}+\mathrm{i}$ 2013-16).

${ }^{2}$ Grevisse (2011 : §1106) considère que oui, non et si en français moderne sont des «mots-phrases » qui ont la valeur d'une phrase antérieure, de la même façon qu'un « pro-nom » équivaut à un nom auquel il renvoie de façon anaphorique. Pour Tesnière (1988: 212), oui et non sont des " mots conventionnels » anaphoriques, c'est-à-dire « des mots vides qui se remplissent automatiquement, soit au positif, soit au négatif, selon les cas, du contenu de l'interrogation à laquelle ils répondent ». Tesnière voit la réponse si comme une variante de oui pour répondre à une interronégative. Ces catégorisations sont sans doute à remettre en cause dans la mesure où, dans certains cas, ces adverbes ne reprennent aucun segment linguistique énoncé explicitement auparavant. Le concept d'《 anaphore » tel qu'on l'utilise habituellement ne permet pas toujours de décrire le fonctionnement de oïl et nennil, et de oui, non, si. Pour des remarques intéressantes à ce sujet voir Hankamer \& Sag (1976), Plantin (1978) et Bosque Muñoz (1984).
} 
marqueurs enchaînent sur un segment de dis cours que le même locuteur a prononcé avant. L'évolution de oïl et nennil et de si ferai je, non est, etc. aboutit en français moderne au système unique de trois adverbes oui/non/si en usage encore aujourd'hui. Le fonctionne ment de oui/non/si en français moderne a été décrit dans le cadre des interactions verbales par Kerbrat-Orecchioni (2001) et dans une perspective sémantique par Plantin (1978, 1982).

Loin de suivre le schéma simpliste traditionnel selon lequel oui marque l'accord avec un énoncé positif, si le désaccord avec un énoncé négatif et non l'accord lorsqu'il enchaîne sur un énoncé négatif et le désaccord lorsqu'il enchaîne sur un énoncé positif, les adverbes oui, non et si du français moderne présentent des distributions différentes en fonction de l'énoncé sur lequel ils enchaînent :

(1) $\mathrm{x}$ : - Ce mur n'est pas blanc.

Y: - Oui ! (en effet) (il n'est pas blanc).

$Y^{\prime}$ : -Non! (en effet) (il n'est pas blanc).

Y" : -(Mais) si ! (il est blanc !).

En reprenant l'exemple classique ce mur n'est pas blanc, Plantin (1982) montre qu'en réponse à une assertion négative oui et non marquent l'accord avec l'allocutaire. Cependant, le fonctionnement sémantique de oui et de non est différent : oui asserte non $\mathrm{p}$, alors que non réfute p (Plantin, 1982 : 263). Seul l'adverbe si marque toujours le désaccord, il réfute non $p$.

L'enchaînement sur un énoncé interrogatif est également complexe. On considère souvent que oui, non et si constituent la réponse uniquement à des interrogations totales. Or, KerbratOrecchioni apporte des exemples tirés d'interactions réelles où non enchaîne sur une interrogation partielle (2001 : 98) :

(2) A1 - le directeur est à Paris pendant toute la semaine donc euh si vous voulez me donner vos coordonnées

B1 - oui c'est monsieur comment le directeur?

A2 - non c'est madame

Dans ce cas, non sert à contester non pas un contenu asserté mais le présupposé « le directeur est un homme ». Plantin signale par ailleurs qu'il existe des interrogations syntaxiquement totales mais discursivement partielles (1982: 259-261):

(3) X : - Voudrais-tu faire quelque chose $\uparrow$

Y: -Oui, manger des noix.

La seule réponse oui ne satisfait pas $\mathrm{X}$. La question ne porte pas sur le «vouloir» de $\mathrm{Y}$, la question sert en vérité à réaliser une offre. Oui répond à l'offre et manger des noix à l'interrogation partielle. Pour Plantin, ce type d'enchaînements met en relief la nature nonanaphorique des adverbes oui, non et si (cf. note 2). Le travail sur un corpus d'interactions orales de Kerbrat-Orecchioni révèle que la plupart des questions sont orientées ${ }^{3}$ (2001 : 109). La polarité de la question et son orientation conditionnent les réponses possibles. Par exemple, une interrogation positive d'orientation négative n'admet pas oui comme marqueur d'accord :

(4) es-tu vraiment partisan de l'euthanasie $\uparrow$ - oui

(5) est-ce que les plantes qui sont autour de lui poussent $\uparrow$-ah si au contraire madame

Dans les exemples (4) et (5) de Kerbrat-Orecchioni (2001 : 110), les questions ont une orientation négative, autrement dit, le locuteur asserte le point de vue négatif sous-jacent « tu n'es pas

\footnotetext{
${ }^{3}$ Pour une étude sur le statut illocutoire des énoncés interrogatifs, voir le chapitre « L'acte illocutoire de question vu à travers les réponses directes » de Diller (1984 : 65-98) et pour une étude en détail sur la demande de confirmation dans les questions orientées voir Borillo (1979).
} 
partisan de l'euthanasie » et « les plantes qui sont autour de lui ne poussent pas » respectivement. D'un point de vue pragmatique oui et si marquent tous les deux le désaccord avec le premier locuteur. D'un point de vue sémantique leur fonctionnement est divergent : oui privilégie la forme de l'interrogation et reprend sa polarité positive, alors que si s'oppose à l'orientation négative de l'interrogation et marque donc la contradiction positive. Ici, la réponse négative non marque rait l'accord avec l'orientation négative de la question.

Nous n'irons pas plus loin dans l'exposition de la complexité des enchaî nements où apparaissent oui, non et si en français moderne. Les particularités sémantico-pragmatiques de ces adverbes proviennent des différentes valeurs de oïl et nennil et des expressions de type si ferai je, non est il, si a, non fait du français médiéval. La description de l'évolution de ces structures depuis l'ancien français jusqu'au français classique tiendra forcément compte du contexte discursif.

Dans cet article nous allons présenter l'évolution des marqueurs avec un verbe substitut de type si ferai je, non est il, si a, non fait, etc. depuis la fin du moyen français (milieu du XVI siècle) jusqu'à la fin du français classique (fin du XVIII ${ }^{\mathrm{e}}$ siècle). Nous commencerons par rappeler le fonctionnement syntaxique, sémantique et pragmatique de ces expressions en ancien et en moyen français (842-1330 et 1330-1550, respectivement). Nous décrirons ensuite les variations que subissent ces marqueurs en français préclassique et classique (1550-1650 et 1650-1789 respective). Nous décrirons ensuite les variations que subissent ces marqueurs en français préclassique et classique (1550-1650 et 1650-1789 respectivement.

Pendant cette période apparaissent de nouvelles variantes dérivées de si ferai je, non est, etc. : les marqueurs oh ! que si/oh ! que non, qui foisonnent dans le théâtre dès la fin du XVII ${ }^{\mathrm{e}}$ siecle, le marqueur redupliqué non, non, ou l'expression figée si fait, qui se réduira postérieurement au si de contradiction du français moderne. Toutes ces variantes surgissent à des moments différents et elles perdurent plus ou moins longtemps dans la langue orale.

Notre étude s'appuie sur un corpus de 2671 occurrences des expressions de type si ferai je, non est il, si a, non fait et de leurs variantes postérieures. Le tableau 1 présente le détail du nombre d'occurrences analysées. La Base de Français Médiéval a fourni la plupart des occurrences d'ancien et de moyen français. Les occurrences de français préclassique et classique, ainsi que quelques-unes de moyen français, proviennent de la base Frantext. Il faut ajouter à cela un certain nombre d'occurrences provenant de dépouillements faits à la main.

Tableau 1

\begin{tabular}{|l|c|c|c|c|c|}
\hline & $\begin{array}{c}\text { Ancien fr. } \\
842-1329\end{array}$ & $\begin{array}{c}\text { Moyen fr. } \\
1330-1549\end{array}$ & $\begin{array}{c}\text { Fr. } \\
\text { préclassique } \\
1550-1649\end{array}$ & $\begin{array}{c}\text { Fr. classique } \\
1650-1789\end{array}$ & Total \\
\hline $\begin{array}{l}\text { si/non + verbe } \\
\text { substitut (+Sp) }\end{array}$ & 231 & 174 & 97 & 66 & 568 \\
\hline $\begin{array}{l}\text { si en emploi } \\
\text { absolu }\end{array}$ & - & 4 & 9 & 22 & 35 \\
\hline $\begin{array}{l}\text { oh/ah/eh ! que } \\
\text { si/non }\end{array}$ & - & - & 1 & 73 & 74 \\
\hline non, non & - & 13 & 743 & 1238 & 1994 \\
\hline Total & 231 & 191 & 850 & 1399 & 2671 \\
\hline
\end{tabular}




\section{SI FERAI JE, NON FAIT EN FRANÇAIS MEDIEVAL}

Dans cette partie, nous nous contenterons d'une présentation succincte des principales caractéristiques des expressions si ferai je, non est il, si a, non fait, etc. en français médiéval, que nous avons exposées dans Saiz-Sánchez (2016).

Les structures de type si ferai je, non est il, si a, non fait de l'ancien et du moyen français enchaînent avec des énoncés assertifs, interrogatifs ou injonctifs. Ce sont des structures fixes dont le fonctionnement ne varie pas au cours de cette période. Les adverbes si et non occupent la première position et ils sont suivis d'un verbe substitut qui reprend les traits syntaxiques d'un énoncé E1 antérieur. Le sujet pronominal (Sp) apparaît postposé au verbe lorsque l'expression marque l'accord avec E1 (voir si fera il en (9)) et il est omis si l'expression marque le désaccord (voir non seroit en (6)). On peut schématiser ces structures avec la formule si/non + verbe substitut $(+\mathrm{Sp})$. Aucun autre élément ne s'intercale entre ces composants. Si l'énoncé E1 comporte les verbes estre et avoir, employés dans leur sens premier ou en tant qu'auxiliaires, les structures si/non + verbe substitut (+ Sp) utilisent le même verbe de E1 comme verbe substitut. Les verbes modaux pooir, vouloir et devoir peuvent jouer également le rôle de verbe substitut lorsqu'ils apparaissent en E1, mais uniquement en ancien français :

(6) S'il savoient qu'il m'ont fait endurer,

Lor falsetez en seroit, espoir, maindre :

Non seroit, voir, trop me vuelent grever.

(R. de Beaujeu, Bel Inconnu, avant 1214, Chanson III, v. 19-21)

[S'ils savaient ce qu'ils m'ont fait subir, leur fausseté serait, peut-être, moindre. Mais non ! Hélas ! ils me veulent trop blesser. (trad. Michèle Perret \& Isabelle Weill)]

(7) Si li cria : «Vasax, por coi

M'avez feru, dites le moi,

Qant devant moi ne vos savoie,

Ne rien mesfet ne vos avoie?

-Par foi, si aviez, fet cil ;

(Chrétien de Troyes, Chevalier de la Charrette ou Lancelot, ca. 1177-1181, v. 773-781)

[« Vassal, lui crie-t-il, pourquoi m’avez-vous frappé, dites-le moi, alors que je ne vous savais pas devant moi et que je ne vous avais causé aucun tort ? Ma foi, si, c'est bien ce que vous avez fait! répondit-il. [...]». (trad. Catherine Croizy-Naquet)]

(8) Gorvains Cadrus tot erroment

Respont ; « Sire compains, por qoi ?

Il m'est avis, si com ge croi,

S'ele ert deable par dedens

Grue, fantosmë ou serpens,

Por la biauté qui est defors

Doit l'en amer dedenz son cors.

- Non doit ! -Si doit, ce m'est avis ».

(R. de Houdenc, Meraugis de Portlesguez, 1225-1235, v. 504-511)

[Gorvain Cadrus répondit vivement : «Pourquoi, mon ami ? À mon avis, -j'en suis convaincu-, même si dans son corps elle avait un diable, une grue, une créature enchantée ou un serpent, par sa beauté extérieure on devrait aimer ce qui est caché dedans. -Non ! -Si, à mon avis!»]

L'exemple (6) est un monologue où le locuteur revient sur une assertion qu'il a énoncée précédemment pour rectifier sa pensée. L'énoncé E1 lor falsetez en serait, espoir, maindre

\footnotetext{
${ }^{4}$ Sauf indication contraire, toutes les traductions sont de nous.
} 
correspond au point de vue positif d'un premier énonciateur ${ }^{5}$ avec lequel le locuteur s'identifie. Mais juste après, en disant non serait, le même locuteur met en scène un nouvel énonciateur qui reprend et inverse la polarité du premier point de vue pour s'y opposer. Non serait marque la contradiction négative avec le point de vue positif du premier énonciateur. Le sujet pronominal n'est pas exprimé après le verbe puisque non serait marque le désaccord avec l'énoncé E1.

Dans les dialogues en (7) et en (8), les structures si aviez, non doit et si doit ne comportent pas non plus de sujet pronominal. Elles marquent également le désaccord, mais cette fois-ci dans l'interaction entre deux locuteurs. En (7), le verbe de si aviez reprend le temps de l'auxiliaire a voir de l'énoncé rien mesfet ne vos a voie. La première personne n'est pas reprise dans la mesure où le mode de désignation d'un même référent varie en fonction de la situation d'énonciation. En tout cas, le référent du sujet du verbe de E1 (je) est le même que celui du verbe de la réponse réfutative (tu). L'adverbe si inverse la polarité négative de E1. En (8), l'expression non doit, contredit l'assertion positive por la biauté qui est defors/ doit l'en amer dedenz son cors. Le verbe modal devoir de E1 est repris par le même verbe dans la réponse et la polarité est inversée avec l'adverbe non. Dans le deuxième tour de parole du premier locuteur, si doit s'oppose à non doit. La structure de ces deux marqueurs est identique mais de polarité inverse. Non doit marque la contradiction négative et si doit la positive.

Lorsque l'énoncé repris contient un verbe autre que estre, a voir ou l'un des trois verbes modaux (uniquement en ancien français), c'est faire qui apparaît dans la réponse confirmative ou réfutative :

(9) « Sire chevaliers, fet il, vos iroiz par nostre conseil a danz abé, car ce est li plus preudons de ceenz et de clergie et de bone vie. -Sire, fet Boorz, por Dieu menez $m$ 'i. » Et cil dit que si fera il volentiers. (Queste del saint Graal, 1225 ou 1230, col. 203d, 1. 5-12)

[« Seigneur chevalier, vous irez sur notre conseil chez monseigneur l'abbé, car il est ici le plus sage pour les choses spirituelles comme pour les choses de la vie. -Seigneur, dit Bohort, au nom de Dieu, menez-moi à lui. » Et cet homme dit qu'il le fera volontiers. (trad. Isabelle Vedrenne)]

Contrairement à (6), (7) et (8), en (9), la structure si fera il apparait dans un discours rapporté indirect. La structure reprend et confirme l'énoncé injonctif de polarité positive menez m'i. La polarité positive est maintenue et le sujet pronominal est exprimé après le verbe : si fera il marque l'accord avec E1.

Le système de marqueurs si/non + verbe substitut $(+\mathrm{Sp})$ présente en français médiéval un fonctionnement argumentatif qui lui permet de confirmer ou de contredire les contenus implicites d'un discours antérieur. Cette propriété, visible dans les enchaînements avec un énoncé assertif ou interrogatif, distingue les marqueurs de type si ferai je, non suis des marqueurs oïl et nennil. Ces derniers constituent la réponse à de "vraies » questions, en d'autres termes, des interrogations totales sur la réponse des quelles le locuteur n'a aucun à priori (Diller, 1984 : 105). L'interrogation met oïl et nennil sur une balance neutre. En revanche, les structures si/non + verbe substitut $(+\mathrm{Sp}$ ) enchaînent sur des énoncés interrogatifs dont la réponse positive et la réponse négative ne sont pas symétriques. Ce sont des questions orientées, rhétoriques ou non, qui comportent ce qu'Anscombre \& Ducrot appellent dans la théorie de l'argumentation une assertion préalable (1983 : 131). Lorsqu'un locuteur L pose la question Est-ce que p ?, il « fait entendre un énonciateur L' qui affirme/a affirmé/pourrait affirmer que $\mathrm{p} »$. $\mathrm{P}$ correspond à l'assertion préalable sous-jacente à l'interrogation.

\footnotetext{
${ }^{5}$ Nous employons ici les termes locuteur et énonciateur dans le sens de la théorie de la polyphonie de Ducrot (1984). Le locuteur est le producteur responsable de l'énoncé, c'est l'être de discours qui dit je. Le locuteur met en scène dans son énoncé un ou plusieurs énonciateurs qui présentent différents points de vue dont le locuteur n'est pas forcément l'origine. Ce locuteur peut donner son accord, s'identifier ou s'opposer à ces différents points de vue.
} 
Ainsi, les expressions si ferai je, non est il, si a, non fait, etc. peuvent renvoyer aux contenus implicites d'une interrogation, tels qu'une assertion préalable ou un présupposé. La réponse si/non + verbe substitut $(+\mathrm{Sp})$ n'apporte aucune information nouvelle à l'allocutaire, elle marque l'accord ou le désaccord avec l'opinion de celui-ci par rapport à quelque chose.

En (7), l'énoncé E1 por coi/ m'avez feru, [...],/ qant devant moi ne vos savoie,/ ne rien mesfet ne vos avoie? est une interrogation partielle qui impose une réponse apportant une nouvelle information. Le locuteur 2 ne peut pas répondre oïl ou nennil puisqu'il ne s'agit pas d'une «vraie » question totale. Le marqueur si aviez ne renvoie pas à l'interrogation por coi/ m'avez feru, il renvoie au présupposé " je ne vous ai fait aucun tort ", rattaché à la proposition subordonnée ne rien mesfet ne vos avoie. Seule une expression du type si/non + verbe substitut (+ Sp) peut enchaîner sur une telle interrogation, mais uniquement pour confirmer ou réfuter un contenu implicite rattaché à celle-ci.

Le comportement des structures de type si/non + verbe substitut $(+\mathrm{Sp})$ avec les énoncés assertifs est le même. Ces structures peuvent renvoyer à l'orientation argumentative d'une assertion, implicite ou explicite. Dans l'enchaînement sur les énoncés assertifs ou interrogatifs, si/non + verbe substitut (+ Sp) marque très majoritairement le désaccord (voir (6), (7) et (8)). Lorsque la structure enchaîne sur un énoncé injonctif, elle marque l'accord et le désaccord dans proportions à peu près équivalentes.

L'évolution des structures de type si ferai je, non est il, si a, non fait, etc. tendra dès le moyen français vers la spécialisation en marqueurs de contradiction. À partir du français préclassique (1550-1650), ces structures marquent le désaccord dans la quasi-totalité des occurrences.

\section{FRANÇAIS PRECLASSIQUE ET CLASSIQUE : DES MARQUEURS D'OPPOSITION}

Dès le dernier quart du XVI ${ }^{e}$ siècle, les expressions si/non + verbe substitut marquent uniquement l'opposition à un discours antérieur. Les structures de type si ferai je, non est il, où le sujet se trouve postposé au verbe, ne permettent plus de confirmer un énoncé antérieur comme elles le faisaient en français médiéval. En français préclassique et classique, les rares occurrences de ces marqueurs suivis du sujet pronominal sont des équivalents des structures médiévales aussi + verbe substitut + sujet (pronominal ou nominal), décrites par Prévost (1999 : 358), que l'on traduit en français moderne par moi aussi ou moi non plus ${ }^{6}$ :

(10) AYMON

Le party me plaist fort.

BEATRIX

Aussi fait-il à moy.

AYMON

J'en suis tout transporté.

BEATRIX

\footnotetext{
6 Les expressions de type si/non + verbe substitut + sujet avec la valeur de moi aussi, moi non plus existent en français médiéval. Ces structures présentent plusieurs différences par rapport à celles de notre étude : le sujet peut être nominal ou pronominal, d'autres arguments peuvent apparaître et il peut ne pas y avoir co-référence entre le sujet de E1 et celui de la reprise (Prévost, 1999 : 361). Dans l'exemple ci-dessous, la structure non + verbe substitut $+\mathrm{Sp}$ comporte en plus un complément régime :

« Sire cevaliers, je ai non Lanselos du Lac et sachiés k’il ne m’est point de bel que vous mon non savés. -Si m’aït Diex, fait li cevaliers, non est il a moi meïsmes. [...]» (Tristan en prose, après 1240, p. 132)

[Seigneur chevalier, mon nom est Lancelot du Lac et sachez qu'il ne me plait point que vous le sachiez. -Par Dieu, fait le chevalier, à moi non plus.]
} 
Si suis-je par ma foy. (R. Garnier, Bradamante, 1585, acte I, scène II)

Comme nous l'avons vu en (7), en français médiéval il y a co-référence entre le sujet de E1 et celui de la reprise confirmative ou réfutative. Mais ce n'est pas le cas dans l'exemple (10) du français préclassique : le référent du pronom je dans si suis-je n'est pas le même que celui du je dans j'en suis transporté. L'expression si suis-je ne renvoie pas à la totalité de l'énoncé j'en suis transporté, elle ne confirme pas E1. L'expression reprend uniquement le segment « être transporté », que Béatrix prend en charge à son tour. Béatrix exprime quel est son sentiment à elle par rapport à la situation qui transporte Aymon. Une glose de la réponse serait «moi aussi je suis transportée ». Si suis-je présente une structure équivalente de l'expression aussi fait-il à moy. La valeur confirmative de si/non + verbe substitut + Sp présentée ci-dessus n'existe en français préclassique et classique que dans des textes archaïsants.

\section{VARIATIONS DANS L'EVOLUTION DE SI FERAI, NON SUIS A PARTIR DU FRANÇAIS PRECLASSIQUE}

À partir du milieu du $\mathrm{XV}^{\mathrm{e}}$ siècle, les marqueurs d'opposition de type si ferai, non suis du français médiéval subissent divers types de variations. Les expressions si + verbe substitut et les expressions non + verbe substitut évoluent de façon différente en français préclassique et classique. Celles qui commencent par si donneront naissance aux marqueurs si fait, oh ! que si et si en emploi absolu, et celles qui commencent par non donneront naissance aux marqueurs non, non et oh! que non.

Les variantes issues des marqueurs de désaccord si ferai, non suis, etc. peuvent être classées en trois catégories :

1. la forme figée si fait

2. le marqueur rédupliqué non, non

3. les adverbes si et non employés sans verbe substitut après une expression de type il dit que, qui donneront lieu par la suite à l'adverbe si en emploi absolu

4. les expressions oh! que si/oh ! que non à caractère expressif qui ne comportent pas de verbe substitut.

Les changements qui aboutissent à ces trois catégories de marqueurs ne se donnent pas simultanément. Même si tous ces nouveaux marqueurs de contradiction coexistent en français classique, ils présentent des particularités et des degrés de grammaticalisation différents.

\section{1. Si fait : un nouveau marqueur de contradiction figé}

Le marqueur d'opposition si + verbe substitut se fige à partir du français préclassique en la forme si fait qui survit dans certains textes $\mathrm{du} \mathrm{xx}^{\mathrm{e}}$ siècle. Le figement se produit à deux niveaux. Premièrement le verbe faire, qui en français médiéval reprenait tous les verbes autres que estre, avoir et les verbes modaux (en ancien français), s'impose aux autres verbes substituts. En français préclassique, dans certains cas, les verbes être et a voir en E1 sont également repris par faire, qui à partir du deuxième tiers du XVII ${ }^{\mathrm{e}}$ siècle, sera le seul verbe substitut.

(11) JULIEN

C'est bien à cest'heure qu'il fault

Se presenter à un assault

Et, qui plus est, tost s'avancer.

L'ADVOCAT

Je voy bien que tu veux gosser.

JULIEN

Gosser, Monsieur ? Non fay, par Dieu, 
Car moy-mesme je viens du lieu

Où il y en a d'estonnez. (J. Grévin, Les Esbahis, 1562, acte V, scène III, p. 204)

(12) Pour l'impossible, continua-t-il, c'est de le faire comme ces dames ; ce sont des oracles au dela de nostre imitation, et au dessus de nos forces. Je ne crois pas qu'il soit possible de deviner comme elles. Si-fait, si-fait, repliqua brusquement Gelaste, je devineray tout aussi bien qu'elles sur certains faits concernant les filles, car elles ne sont pas encore rafinées.

(M. de Pure Abbé, La Prétieuse ou le Mystère des ruelles, parties 1-2, 1656, Première Conversation, p. 341-342)

En (11), l'interrogation de Julien gosser, Monsieur ? reprend l'assertion antérieure tu veux gosser pour la remettre en question. La réponse non fay marque le désaccord avec l'assertion. Ce marqueur de contradiction est construit avec le verbe faire pour reprendre un énoncé qui comporte le verbe modal vouloir. En ancien français, la reprise réfutative aurait conservé le verbe modal, on aurait trouvé non voil. En (12), l'énoncé je ne crois pas qu'il soit possible de deviner comme elles correspond à une structure je ne crois pas que p qui équivaut a non $\mathrm{p}$. Le marqueur si-fait s'oppose a non p, «il n'est pas possible de deviner comme ces dames ». Le verbe faire reprend le verbe être de l'assertion antérieure.

Le tableau 2 présente la fréquence des marqueurs si/non + être, si/non +avoir et si/non + faire en français préclassique et classique dans notre corpus.

Tableau 2

\begin{tabular}{|l|c|c|c|c|}
\hline \multirow{2}{*}{} & \multicolumn{2}{|c|}{ Français préclassique } & \multicolumn{2}{c|}{ Français classique } \\
\cline { 2 - 5 } & si & non & si & non \\
\hline être & 16 & 20 & - & - \\
\hline avoir & 14 & 5 & - & 3 \\
\hline faire & 50 & 47 & 63 & - \\
\hline
\end{tabular}

On observe qu'en français classique faire est le seul verbe substitut. Par ailleurs, il faut signaler que le marqueur de contradiction positive si + faire est largement majoritaire par rapport à celui de contradiction négative non+ faire, qui n'apparaît que 3 fois dans notre corpus.

Le deuxième niveau de figement correspond au figement du verbe faire à la troisième personne du singulier du présent de l'indicatif indépendamment des traits syntaxiques du verbe de l'énoncé repris.

(13) ANGELIQUE, d'un air languissant.

Est-ce que vous aviez quelque dessein pour elle?

MAITRE BLAISE

Oui, je n'en fais pas le fin.

ANGELIQUE, languissante.

Sur ce pied-là, vous ne m'aimez pas.

MAÎTRE BLAISE

$\mathrm{Si}$ fait da : ça m'avait un peu quitté, mais je vous r'aime chèrement à cette heure. (Marivaux, L'épreuve, 1740, p.541, scène XIX)

(14) MONSIEUR DE POURCEAUGNAC

Est-ce que vous n'êtes pas de mes amis?

SBRIGANI

Si fait; on ne peut pas l'être davantage.

(Molière, Monsieur de Pourceaugnac, 1670, p. 296, acte II, scène IV)

En (13), la réponse si fait de Maître Blaise marque le désaccord avec l'assertion d'Angélique 
vous ne m'aimez pas. Le verbe faire n'est plus conjugué à la première personne du singulier (si fais), l'expression se trouve donc déjà figée. Il en est de même en (14). L'expression si fait marque l'opposition avec le segment vous n'êtes pas de mes amis. Si les règles de la langue médiévale avaient été respectées, la reprise comporterait le verbe être à la première personne du singulier. La réponse serait si suis et non pas si fait.

Le tableau 3 présente en détail les différents types d'enchaînements de si/ non + faire en français préclassique et classique de notre corpus. La ligne 1 correspond aux enchaînements où la structure si/non + faire n'est pas figée, elle suit les règles de reprise de la langue médiévale. Le verbe de l'énoncé E1 n'est pas à la troisième personne du singulier du présent de l'indicatif et l'expression si/non + faire reprend la personne et le temps de E1. Nous aurons donc des réponses du type si fera, si ferions, non feray ou non fay et non pas si fait ou non fait. Nous avons un exemple en (11) où non fay s'oppose à tu veux gosser. La ligne 2 présente la fréquence des expressions si/non fait que l'on considère figées, autrement dit, la structure reprend un énoncé avec un verbe conjugué à une personne autre que la troisième du singulier et/ou à un temps autre que le présent de l'indicatif. C'est ce que nous avons vu en (13) et en (14) où si fait marquait l'opposition avec vous ne m'aimez pas et avec vous n'êtes pas de mes amis, respectivement. Enfin, la ligne 3 correspond aux enchaînements sur le figement desquels nous ne pouvons tirer aucune conclusion, puisque l'expression si/non fait reprend un énoncé $\mathrm{E} 1$ avec un verbe déjà à la troisième personne du singulier du présent de l'indicatif, comme c'était le cas en (12) : si-fait contre dit il n'est pas possible de deviner comme elles. Nous incluons cependant ces données sur le tableau 3 pour que le chiffre total d'expressions si/non + faire corresponde à celui des tableaux 1 et 2 .

Tableau 3

\begin{tabular}{|c|c|c|c|c|c|c|}
\hline & & \multicolumn{2}{|c|}{ Français préclassique } & \multicolumn{2}{|c|}{ Français classique } & \multirow[t]{2}{*}{ Total } \\
\hline \multicolumn{2}{|r|}{ Types d'enchaînements } & si & non & si & non & \\
\hline 1 & $\begin{array}{l}\text { Réponse si/non + faire non figée } \\
\text { E1 : Je voy bien que tu veux gosser } \\
\text { Réponse : Non fay }\end{array}$ & 33 & 40 & 2 & 3 & 78 \\
\hline 2 & $\begin{array}{l}\text { Réponse si/non fait figée } \\
\text { E1: Vous ne m'aimez pas } \\
\text { Réponse : Si fait }\end{array}$ & 14 & 6 & 55 & - & 75 \\
\hline 3 & $\begin{array}{l}\text { Réponse si/non fait non pertinente } \\
\text { E1 : Je ne crois pas qu'il soit possible } \\
\text { de deviner comme elles } \\
\text { Réponse : Si-fait }\end{array}$ & 3 & 1 & 6 & - & 10 \\
\hline \multirow{2}{*}{\multicolumn{2}{|c|}{ Total }} & 50 & 47 & 63 & 3 & \multirow[t]{2}{*}{163} \\
\hline & & \multicolumn{2}{|c|}{97} & \multicolumn{2}{|c|}{66} & \\
\hline
\end{tabular}

La ligne 2 du tableau 3 montre que le figement de si fait se produit pendant la période classique : 55 des 66 occurrences de si/non + verbe substitut en français classique, soit plus de $80 \%$, ont la forme si fait indépendamment de l'énoncé avec lequel elles marquent le désaccord, alors qu'en français préclassique seul 14 des 97 occurrences sont figées, soit moins de 15\%. Le français préclassique constitue une période de transition où le verbe faire peut encore reprendre les traits syntaxiques de E1.

Par ailleurs, les expressions si/non + verbe substitut changent de niveau de langue. Parmi les dictionnaires et les grammaires anciennes que nous avons consultés ${ }^{7}$, la $1^{\text {re }}$ édition du Dictionnaire de l'Académie française (1694) est le premier ouvrage à considérer ces expressions des «façons de parler basses », termes repris par le Dictionnaire universel françois et latin de

\footnotetext{
${ }^{7}$ Nous avons examiné les 14 dictionnaires et grammaires en version électronique du Grand atelier historique de la langue française (2003), qui datent de 1606 à 1876. Nous avons également consulté les Remarques sur la langue françoise de Vaugelas (1647) et la Grammaire et syntaxe françoise de Maupas (1625), où rien n'est mentionné au sujet du niveau de langue de si fait.
} 
Trévoux (1743-1752). La $4{ }^{\text {ème }}$ édition du Dictionnaire de l'Académie française (1762) qualifiera si fait de «façon de parler familière \& qui n'est pas du bel usage ». Le Dictionnaire critique de Féraud (1787) en fera autant quelques années plus tard. Les autres ouvrages consultés ne signalent rien à ce sujet, à l'exception de Callières (1693), comme nous allons le voir ci-dessous.

Ainsi, ces marqueurs caractérisent souvent le discours de certains personnages du thêâtre du français classique : bourgeois, valets, paysans, suivantes, etc. C'était le cas en (14), où Sbrigani est un personnage bourgeois. Mais si fait est également employé par des personnages d'une classe sociale plus élevée pour marquer la contradiction forte. En (13), le marqueur si fait permettait à Maître Blaise de s'opposer frontalement au discours d'Angélique. Dans Du bon et du mauvais usage dans les manières de s'exprimer (1693), Callières signale à propos de l'usage de ces expressions :

Il y en a qui disent souvent en conversation, si fait, non fait $^{8}$; ces termes me paroissent durs \& mal polis ; il faut dire oüi \& non, ou vous m'excuserez, vous me pardonnerez, si je vous dis que cela est, ou que cela n'est pas ainsi, \& d'autres adoucissements de cette sorte, lorsqu'on est obligé de contredire quelqu'un. (1693: 146-147)

Pour Callières, il n'est pas question de niveau de langue, il est question de politesse. Les structures si fait et non fait sont des réponses non-préférées dans la mesure où elles marquent le désaccord là où l'allocutaire cherche $1^{\text {'accord }}{ }^{9}$. Callières rend compte au XVII ${ }^{\mathrm{e}}$ siècle de ce que le linguiste Pomerantz expliquera trois siècles plus tard : dans les actions qui invitent à l'accord, l'allocutaire essaye d'éviter ou de retarder au maximum l'expression du désaccord en ayant recours, entre autres, à des demandes de clarification ou à des expressions affaiblissant l'opposition (1984: 7076). Si fait serait donc une réponse non-préférée en raison de sa valeur d'opposition forte.

\section{2. Non, non : un nouveau marqueur rédupliqué}

Les chiffres du tableau 3 montrent également que, s'il est vrai que la fréquence de si fait figé (ligne 2) augmente considérablement entre les deux périodes (on passe de 14 à 55 occurrences), la fréquence de non + faire chute : en français préclassique non + faire apparaitt 47 fois dans notre cor pus et en français classique il n'apparaît que 3 fois. Face au déclin de non + faire, à partir du français préclassique le marqueur non, non (écrit aussi non non sans virgule) prend le relais pour exprimer la contradiction négative. Dans les textes médiévaux on trouve des occurrences des expressions de type non ferez, non, non, où l'adverbe non est répété après le verbe. Il est rare que non soit répété sans être immédiatement suivi d'un verbe : 12 des 13 occurrences de non, non datent en plus de la fin du moyen français. En revanche, pour la période 1550-1649, Frantext apporte 743 occurrences de non, non et pour la période 1650-1789, 1238 occurrences.

Dans ses travaux sur les «multiple sayings », Stivers explique que la répétition ou réduplication de mots ou groupes de mots est « a communication practice in its own right and may be understood as analyzably separate from the item being repeated » (2004: 269). En français préclassique, le marqueur rédupliqué non, non a une valeur différente de celle du marqueur non sans rédupliquer, qui avait déjà un emploi absolu en français médiéval ${ }^{10}$. De même que si/non + verbe substitut en français médiéval, non, non en français préclassique et classique permet au locuteur de réfuter les contenus implicites d'un discours antérieur. Le marqueur non, non ne s'oppose pas à un énoncé concret, il s'oppose à un contenu sous-jacent, voire même à la

\footnotetext{
${ }^{8}$ Les italiques sont dans le texte d'origine.

${ }^{9}$ Nous nous appuyons sur l'analyse de l'interaction anglo-saxonne. Voir, par exemple, le chapitre « The organization of preference/dispreference » dans Schegloff (2007: 58-96).

${ }^{10}$ En français médiéval, l'adverbe non en emploi absolu concurrençait le marqueur nennil, dont la fréquence diminue à partir du français préclassique. Frantext apporte 118 occurrences de nenny et ses variantes en français préclassique, et 72 en français classique.
} 
pertinence d'énoncer E1. Le marqueur rédupliqué vise non seulement le tour de parole auquel il répond immédiatement, mais aussi «the larger preceding course of action that the turn embodies » (Stivers, 2004 : 269). Non, non marque le désaccord avec l'énonciation de l'allocutaire en général, comme on le voit en (15) et en (16) :

\section{(15) NABUCHODODOSOR}

Eus-je tort de poursuivre un rompeur d'alliance, Et qui print contre moy d'Egypte l'accointance?

\section{AMITAL}

Non, vous n'eustes pas tort, et non non, ce fut nous,

Nous mesmes de nos maux sommes cause, et non vous. (R. Garnier, Les Juifves, 1585, p. 60, acte III)

Dans cette scène de Les Juifves, Nabuchodonosor accuse Amital de ne pas avoir empêché la trahison du fils de celle-ci pour éviter ainsi les malheurs de leur peuple, le peuple hébreu. Amital se justifie en disant qu'elle avait prévenu son fils des conséquences mais qu'il fut trop arrogant. C'est alors que Nabuchodonosor demande à Amital s'il eut tort d'agir comme il le fit. Dans la réponse d'Amital, le marqueur non sans rédupliquer reprend et contredit la proposition « j'eus tort de poursuivre un rompeur d'alliance, et qui print contre moy d'Egypte l'accointance » qui correspond à l'assertion préalable de l'interrogation de Nabuchodonosor. L'énoncé vous n'eustes pas tort, équivalent de E1 mais de polarité inverse, rend le désaccord explicite. C'est une réponse à une vraie question, ou du moins, Amital l'interprète comme une question neutre, non orientée. En revanche, le marqueur rédupliqué non, non marque le désaccord avec ce que l'on peut inférer de l'interrogation de Nabuchodonosor: « je suis coupable du malheur de votre peuple ». Non, non s'oppose à la conclusion (ou orientation argumentative) visée par le discours de l'allocutaire et non pas à un segment concret comme le faisait non. Amital censure en quelque sorte le fait que son allocutaire produise un discours dont on peut conclure que ce serait lui le coupable du malheur des juifs. L'énoncé ce fut nous / Nous mesmes de nos maux sommes cause, et non vous explicite ce à quoi renvoie le marqueur d'opposition rédupliqué non non.

L'exemple (16) met en relief une autre caractéristique du marqueur rédupliqué :

\section{(16) MEDOR}

Helas ! ay-je pû vous déplaire?

\section{ANGELIQUE}

Non, non, je n'ay point de colere...

Laissons des discours superflus.

Partez. (P. Quinault, Roland, 1685, acte I, scène 4)

Le marqueur non, non ne s'oppose pas à l'interrogation ay-je pû vous déplaire ?. Il s'oppose à la pertinence de celle-ci. Le marqueur enchaîne sur l'énonciation du discours de l'allocutaire, il peut être glosé par « vous avez tort de me poser la question ». L'opposition à la pertinence de la question est renforcée par l'énoncé laissons des discours superflus. Non, non est souvent suivi d'énoncés qui font allusion au dire du discours antérieur plutôt qu'au dit.

Le marqueur non, non du français préclassique correspond à ce que Dostie (2007) appelle une « réduplication lexicale » dans la mesure où il s'agit d'un nouveau marqueur avec une valeur différente de celle du marqueur non qui serait répété uniquement pour renforcer l'opposition ${ }^{11}$.

$\mathrm{Si}$ fait et non, non sont les deux marqueurs qui en français préclassique et classique héritent des

\footnotetext{
${ }^{11}$ Dostie oppose « réduplication lexicale » à « réduplication pragmatique ». La deuxième correspond à la répétition d'une expression dans le but de renforcer sa valeur. Dans ce cas, l'expression rédupliquée n'a pas une valeur différente de celle de l'expression non-rédupliquée. Par ailleurs, Dostie (2007 : 46) explique que le contour prosodique de ces deux types de réduplications n'est pas le même. Stivers le signale aussi : «multiple sayings are done under a single intonation contour» $(2004: 261)$. Malheureusement, ces traits ne peuvent être étudiés pour des états de langue anciens.
} 
valeurs sémantico-pragmatiques des structures si/ non + verbe substitut $(+\mathrm{Sp}) \mathrm{du}$ français médiéval. Certaines variations que l'on voit apparaître déjà en moyen français préfigurent l'emploi de si de façon absolue ainsi que l'apparition de constructions du type oh ! que si/non qui marquent la forte opposition en français classique.

\section{3. De que si/que non à si en emploi absolu}

Dès le moyen français, lorsque les expressions si/non + verbe substitut marquent le désaccord et qu'elles sont introduites par une structure de type je gage que, elle dit que, je suis sûr que etc., le verbe substitut est susceptible d'être omis. Les adverbes si et non suffisent à marquer l'opposition :

(17) Le prince disant que le chevalier de Gramont avait l'autre jour ses poches pleines d'argent, il en prit à témoin La Feuillade, qui dit que cela n'était point et qu'il n'avait pas un sou. « Je vous dis que si. - Je vous dis que non. [...] » $\left(\mathrm{M}^{\mathrm{me}}\right.$ de Sévigné, 1675, Correspondance, p. 36).

En ancien français, les marqueurs de désaccord si et non auraient été suivis d'un verbe substitut. Nous aurions trouvé quelque chose comme je vous dis que si avait et je vous dis que non avait. Mais dès la fin du moyen français, le verbe substitut n'est plus indispensable lorsque l'expression est introduite par un verbe de parole. Même si l'usage de que si/que non sans verbe substitut se répand en français préclassique et classique, les marqueurs de type si/non + verbe substitut continuent à s'employer en dehors des structures complétives.

Etant donné que non sans être suivi d'un verbe substitut existait déjà en français médiéval, il est extrêmement difficile de déterminer si non inséré dans une proposition complétive est le fruit de l'omission du verbe dans non + verbe substitut ou s'il provient de l'emploi absolu de non. Certains indices, dont la présence d'une expression que si dans le contexte proche ou le renvoi à un contenu implicite, permettent éventuellement de considérer qu'il y a une omission du verbe substitut. En tout cas, la construction $\mathrm{p}$ que si constitue la première étape dans l'évolution vers l'emploi absolu du marqueur de contradiction si.

Par ailleurs, à partir du français classique, l'expression que si, que non peut apparaître lexicalisée par le phénomène de dérivation délocutive (Anscombre, 1985) :

(18) Le Renard au Chat dit enfin :

Tu prétends être fort habile :

En sais-tu tant que moi ? J'ai cent ruses au sac.

Non, dit l'autre : je n'ai qu'un tour dans mon bissac,

Mais je soutiens qu'il en vaut mille.

Eux de recommencer la dispute à l'envi.

Sur le que si, que non, tous deux étant ainsi,

Une meute apaisa la noise.

(J. de La Fontaine, Fable XIV Le chat et le renard, Fables, livre 9, 1679, p. 372)

L'expression que si, que non se trouve actualisée au moyen de l'article défini le et se trouve ainsi classé dans la catégorie grammaticale des substantifs et non plus celle des marqueurs du discours $^{12}$. Dans certains contextes, que si, que non acquiert une valeur sémantique nouvelle qui provient de la valeur pragmatique d'opposition lorsque l'expression est énoncée. En d'autres termes, que si, que non a la valeur de l'« acte illocutoire que l'on peut accomplir en énonçant la formule » (Anscombre, 1985 : 24). En (18), le sens de l'expression est celui de « querelle au cours

\footnotetext{
${ }^{12}$ Nous pourrions parler ici de lexicalisation dans le sens de Brinton \& Traugott $(2005: 38)$ : un mot ou un groupe de mots change d'une classe grammaticale mineure à une majeure, il y a un développement « of a fully referencial lexical item from a non lexical or grammatical item ».
} 
de laquelle on énonce que si, que non ».

L'emploi absolu de si en français préclassique et classique est possible en dehors des structures complétives. Le marqueur si était cependant moins répandu que si fait. Notre corpus en français préclassique comporte 9 occurrences de si en emploi absolu et 22 en français classique. En revanche, si fait figé apparaît 14 et 55 fois respectivement (cf. tableau 3). Voici la première occurrence de si en emploi absolu que l'on trouve dans Frantext :

\section{(19) SATAN}

Moy, que je me desiste, et que je vienne au poinct

De ne le plus tenter, et ne poursuivre point?

Non, je ne cesseray, que par toute traverse

La foy qu'il a tant forte en fin je ne renverse.

Ne le pourray-je pas? Si, je le pourray, si.

Si j'ay veincu Adam, je pourray veincre aussi

Les enfans malheureux. (L. De Masures, David fugitif, 1566, p. 198)

Il s'agit d'un emploi monologal où Satan se questionne lui-même sur sa capacité de vaincre la foi chrétienne de David. Le locuteur présente deux énonciateurs qui entretiennent un vrai dialogue. Le premier énonciateur asserte d'abord qu'il ne cessera de lutter jusqu'à ce qu'il n'anéantisse la foi de David. Ensuite, l'interrogation Ne le pourray-je pas ?, attribuée au deuxième énonciateur, remet en question l'assertion antérieure. La réponse si, je le pourray, si, prise en charge par le premier énonciateur, contredit l'assertion préalable à l'interrogation « je ne pourrai pas le vaincre ». L'adverbe si n'est plus suivi du verbe substitut faire : il suffit à marquer le désaccord. Toutefois, l'énoncé je le pourray complète la réponse pour expliciter ce à quoi si s'oppose. Le marqueur si est en plus répété après je le pourray. Ce type de structure, où un verbe qui n'est pas substitut apparaît après le marqueur si, relève en même temps de l'usage ancien et de l'usage moderne de ces expressions réfutatives.

De même que le marqueur non, non rédupliqué, qui a un fonctionnement différent de non, si peut apparaître aussi rédupliqué :

(20) JEAN-LOUIS

Or çà, Louise, il faut que tu fasses ce qu'a recommandé Mme la duchesse.

LOUISE

Quelle fantaisie!

JEAN-LOUIS

Elle le veut; et voilà la lettre.

LOUISE

Vous ne voulez pas nous la lire?

JEAN-LOUIS

$\mathrm{Si}$, si, si, je vais vous la lire : mais il faut bien m'écouter, et ne pas m'interrompre, comme vous faites les soirs, quand je lis de mon gros livre.

(M.J. Sedaine, Le Déserteur, 1769, scène 2, acte I, p. 132)

D’une part, le marqueur rédupliqué si, si, si s'oppose à " vous n'allez pas nous lire la lettre ", orientation argumentative de l'interrogation de Vous ne voulez pas nous la lire ?, et d'autre part, tout comme non, non, il marque l'opposition à l'énonciation de la question. Si, si, si peut être glosé à nouveau par « vous avez tort de me poser cette question ».

En français préclassique et classique l'usage de si en emploi absolu est encore assez réduit. Il faut attendre le français moderne pour le voir s'imposer.

4. 3. Oh! que si et oh! que non :

emploi absolu et renforcement de l'opposition 
La dernière variante des expressions si ferai, non est, etc. correspond aux marqueurs oh ! que si et oh ! que non qui ne comportent pas de verbe substitut et qui ont en plus une valeur d'opposition renforcée.

Les constructions de type oh/ah/eh ! que p apparaissent très souvent en français classique pour emphatiser l'opposition au discours antérieur. Vers la fin $\mathrm{du} \mathrm{XVII}^{\mathrm{e}}$ siècle, les adverbes si et non sans verbe substitut rentreront également dans les constructions interjectives de ce type. Les expressions oh/ah/eh ! que + si/non constituent par elles-mêmes la réponse à un énoncé antérieur. Dans la plupart cas ces marqueurs sont construits avec l'interjection oh suivie d'un marqueur graphique d'exclamation, comme c'est le cas de (21), ou d'une virgule, en (22) :

(21) Eh bien ! Oui, il saura le lieu de ma retraite ; que m'importe ? Qu'en peut-il arriver ? Rien, à ce qu'il me semble. Est-ce qu'il tentera de me voir ou de m'écrire ? Oh! Que non, me disais-je. Oh! Que si, devais-je dire, si je m'étais répondu sincèrement, et suivant la consolante apparence que j'y trouvais. (Marivaux, La Vie de Marianne, partie 3, 1745, p. 159)

(22) SOLIMAN

Oüi ; mais je crois, cher Ali,

qu'elle ne sera pas contente

de mon ardeur trop pressante.

ALI

Oh, que si !

SOLIMAN

Je vais de cette inhumaine

augmenter pour moi la haîne.

ALI

Oh, que nenni !

(A.-R. Lesage, Achmet et Almanzine, 1728, p. 465-466, acte III, scène 3)

Les marqueurs oh ! que si et oh ! que non sont les structures interjectives les plus représentées dans notre corpus en français classique, essentiellement dans le genre théâtral. En français préclassique nous avons trouvé uniquement l'expression oh ! si fais déa. D'un total de 73 structures de type oh/ah/eh! que + marqueur d'opposition en français classique, 53 présentent la forme figée oh ! que si ou oh ! que non, soit presque $75 \%$. Il existe néanmoins d'autres associations d'interjections et de marqueurs d'opposition, comme en témoignent les exemples (23) à (26). Dans certains cas que est omis :

\section{(23) ARLEQUIN}

Êtes-vous bien aise de me voir?

SILVIA

Assez.

ARLEQUIN, en répétant ce mot

Assez! ce n'est pas assez.

SILVIA

Oh si fait, il n'en faut pas davantage.

(Marivaux, Arlequin poli par l'amour, 1723, scène VI, p. 98)

\section{(24) DORANTE}

Je suis persuadé que tu n'es point fâché que je lui en conte.

ROSIMOND

Ah ! si fait ; très-fâché. J'en boude, et si vous continuez, j'en serai au désespoir. (Marivaux, Le Petit-maître corrigé, 1739, p. 202-203, acte II, scène 3)

(25) Il est vrai, lui dis-je, que ces longueurs m'ont quelquefois impa tienté dans les avantures de Théagene, de Cyrus, de Cléopatre, et de plusieurs autres. Mais ne puis-je pas abréger les formalités... eh si, me répondit-il, vous siéroit-il de ne faire qu'un petit chapitre des mille et une nuit, ou des contes chinois. (G.-H. Bougeant, Voyage merveilleux du prince Fan-Férédin dans la 
Romancie, 1735, p. 151, chap. 10)

(26) SUZANNE, raillant

Hélas ! L'heureux bonnet, et le fortuné ruban qui renferment la nuit les cheveux de cette belle marraine...

CHERUBIN, vivement

Son ruban de nuit ! donne-le-moi, mon cœur.

SUZANNE, le retirant

Eh ! que non pas ; - son cœur ! Comme il est familier donc ! Si ce n'était pas un morveux sans conséquence.

(Beaumarchais, Le Mariage de Figaro, 1785, p. 275, acte I, scène 7)

En (23), le marqueur figé si fait précédé de l'interjection oh s'oppose à l'assertion négative ce n'est pas assez. Ce même marqueur est précédé de l'interjection ah en (24). Ah ! si fait réfute de façon énergique l'assertion «tu n'es point fâché que je lui en conte ». L'adverbe si en emploi absolu est précédé en (25) de eh pour marquer le désaccord avec l'assertion préalable à l'interrogation « je ne peux pas abréger les formalités ». Contrairement aux exemples précédents, en (26), l'interjection est accompagnée d'un que exclamatif. L'expression eh ! que vient renforcer le marqueur non pas, seule occurrence dans notre corpus de l'expression oh/ah/eh ! que + non avec le forclusif pas. Ici le marqueur enchaîne sur un énoncé injonctif.

L'interjection dans une structure oh/ah/eh! que renforce d'une part le marqueur d'opposition et d'autre part, comme l'explique Rosier, elle « participe activement de cet effet de réel en mimant la spontanéité du locuteur qui s'exprime » (2000 : 24). En effet, ces marqueurs relèvent de l'oral, même s'ils apparaissent dans des textes écrits, souvent pour être joués. Les auteurs cherchent à représenter une langue orale à travers un medium écrit, qui est tout sauf spontané.

\section{CONCLUSION}

Les constructions du type si/non + verbe substitut $(+\mathrm{Sp})$ du français médiéval manquent l'accord et le désaccord entre locuteurs. Leur fonctionnement pragmatique leur permet de renvoyer à des contenus implicites d'un discours antérieur, pour les confirmer ou les réfuter. En français préclassique et classique ces constructions marqueront uniquement le désaccord et évolueront pour donner d'autres marqueurs plus ou moins figés.

En français médiéval, le verbe substitut variait en fonction de l'énoncé repris. En français classique seul le verbe faire rentre dans ces constructions qui, par ailleurs, se figent en la forme si fait. Le marqueur rédupliqué non, non, inexistant en français médiéval, reprend, à partir du français pré-classique, la valeur pragmatique de si/non + verbe substitut. Non, non s'oppose à des contenus que l'on peut inférer d'un discours antérieur, voire à la pertinence d'énonciation de celui-ci.

Dès la fin du moyen français, les adverbes si et non suffisent à marquer le désaccord lorsqu'ils sont introduits dans une complétive par des expressions comme il dit que, je réponds que, etc. Les structures interjectives oh/ ah/eh ! que du français classique acceptent également ces marqueurs emploi absolu. Les expressions oh ! que si et oh ! que non prolifèrent dans le théâtre du français classique pour marquer une opposition forte.

En dehors de ce type de constructions, l'emploi absolu de si est rare. Le marqueur de contradiction positive le plus répandu en français préclassique et classique est si fait. Ce n'est qu'à partir du $\mathrm{XIX}^{\mathrm{e}}$ siècle que si en emploi absolu s'impose pour compléter le système d'accord et de désaccord oui/non du français moderne. 


\section{BIBLIOGRAPHIE}

Bases de données

BFM - Base de Français Médiéval. Lyon, ENS de Lyon, Laboratoire ICAR, 2013. 〈http:// bfm.ens-lyon.fr〉

Base textuelle FRANTEXT. ATILF - CnRS \& Université de Lorraine. <http://www.frantext.fr>

\section{Dictionnaires et grammaires anciens}

Dictionnaire de l'Académie Française, 1694, $1^{\mathrm{e}}$ édition et 1762, $4^{\mathrm{e}}$ édition. Publiées en ligne par le Projet ARTFL, de l'Université de Chicago et le Laboratoire de Traitement Automatique de la Langue Française du CNRS.

Grand atelier historique de la langue française, CD-Rom, Marsanne, Redon, 2003.

CALLIERES, F., 1693, Du bon et du mauvais usage dans les manières de s'exprimer : des façons de parler bourgeoises, Paris, Claude Barbin.

FERAUD DE L'ABBE J. F, 1787-1788, Dictionnaire critique de la langue française, in Grand atelier historique de la langue française, CD-Rom. Marsanne, Redon, 2003.

MAUPAS, C., 1628, Grammaire et syntaxe françoise. Genève, Slatkine reprints, 1973.

TREVouX, 1743-1752, Dictionnaire universel françois et latin, in Grand atelier historique de la langue française, CD-Rom. Marsanne, Redon, 2003.

VAUGELAS, C. F., 1647, Remarques sur la langue françoise, utiles à ceux qui veulent bien parler et bien escrire. Paris, Vve J. Camusat et P. Le Petit. Disponible sur Gallica <http://gallica.bnf.fr/>.

\section{Études scientifiques}

ANSCOMBRE, J.-C., 1985, « De l'énonciation au lexique : mention, citativité, délocutivité », Langages, 80, p. 934.

Anscombre, J.-C. \& Ducrot, O., 1983, L'argumentation dans la langue. Bruxelles, Pierre Mardaga.

BORILLO, A., 1979, «La négation et l'orientation de la demande de confirmation », Langue française, 44 (1), p. 27-41.

BOSQUE MUÑOZ, I., 1984, « Negación y elipsis », ELUA. Estudios de Lingüística Universidad de Alicante, 2, p. 171-199.

Brinton, L. J., \& TraugotT, E.C., 2005, Lexicalization and language change. Cambridge, Cambridge University Press.

BURIDANT, C., 2001, Grammaire nouvelle de l'ancien français, Paris, SEDES.

CAPIN, D., 2013, « He, ho, ha, dea : interjections, connecteurs ou marqueurs discursifs ? Le témoignage des textes en prose médiévaux », Diachroniques, 3, Marques d'oralité en français médiéval, p. 95-117.

DILLER, A.-M., 1984, La pragmatique des questions et des réponses, Tübingen, Gunter narr Verlag Tübingen.

DostiE, G., 2004, Pragmaticalisation et marqueurs discursifs: analyse sémantique et traite ment lexicographique, Bruxelles, De Boeck-Duculot.

— 2007, «La réduplication pragmatique des marqueurs discursifs. De là à là là », Langue française, 154 (2), p. 45-60.

DuCROT, O., 1984, Le dire et le dit, Paris, Editions de Minuit.

FLORICIC, F., \& Mignon, F., 2007, « Non non et no no en français et en italien : réitération ou réduplication », dans A. Michaud \& A. Morgenstern (éds.), Faits de langues, La réduplication, Paris, Ophrys, p. 49-61.

GougEnHEIM, G., 1974, Grammaire de la langue française du seizième siècle. Paris, A. et J. Picard. 
GREVISSE, M., 2011, Le bon usage : grammaire française 75 ans, $15^{\mathrm{e}}$ éd. (1 ${ }^{\mathrm{e}}$ éd.1936), Bruxelles, Paris, De Boeck-Duculot.

HANKAMER, J. and SAG, I., 1976, « Deep and Surface Anaphora », Linguistic Inquiry, 7 (3), p. 391-428.

HedDesheIMER, C., 1974, « notes sur l'expression verbale de l'assentiment et de la confirmation en anglais », Mélanges pédagogiques du Centre de Recherches et d'Applications Pédagogiques en Langues, p. 29-40.

HugueT, E., 1989, Dictionnaire de la langue française du seizième siècle, Genève, Slatkine.

Kerbrat-ORECCHIONI, C., 1990-1994, Les Interactions verbales, t.I, t.II, t.III, Paris, Armand Colin.

— 2001, «Oui, Non, Si : un trio célèbre et méconnu », Marges Linguistiques, 2, p. 95-119.

MARCHELlo-NizIA, C., 1985, « Si + faire (avoir/estre) », Actes du IV Colloque international sur le moyen français, Amsterdam, Rodopi, p. 201-215.

— 2012, "L' 'oral représenté' en français médiéval, un accès construit à une face cachée des langues mortes », dans B. Combettes, E. Oppermann-Marsaux, A. Lavrentiev, C. Guillot, \& S. Prévost (éds.), Le Changement en français. Études de linguistique dia chronique, Bern, Berlin, Bruxelles, Peter Lang, p. 247-264.

Plantin, C., 1978, Oui, non, si - Etude des enchaînements dans le dialogue, Thèse de Doctorat d'Etat, EHESS Université de Paris VIII.

— 1982, «Oui et non sont-ils des pro-phrases » Le Français Moderne, 3, p. 252-265.

POMERANTZ, A., 1984, «Agreeing and disagreeing with assessments: some features of preferred/dispreferred turn shapes », dans J.M. Atkinson \& J. Heritage (éds.), Structures of Social Action, Cambridge, Cambridge University Press, p. 57-101.

Prevost, S., 1999, «'Aussi' en position initiale : évolution sémantico-syntaxique du $12^{\mathrm{e}}$ au $6^{\mathrm{e}}$ siècle », Verbum, XXI (3), p. 351-380.

ROSIER, L., 2000, « interjection, subjectivité, expressivité et discours rapporté à l'écrit : petits effets d'un petit discours », Cahiers de praxématique, 34, p. 19-50.

SAIZ-SÁNCHEZ, M., (2016), «Si ferai je, non ferai : l'expression de l'accord et du désaccord dans le dialogue médiéval », Le discours et la langue, 7, Les Discours rapportés en fran çais médiéval au prisme du genre, Fernelmont, EME Editions.

ScheglofF, E.A., 2007, Sequence Organization in Interaction: a Primer in Conversation Analysis, Cambridge, Cambridge University Press.

STIVERS, A., 2004, «'no no no' and Other Types of Multiple Sayings in Social interac tion », Human Communication Research, 30 (2), p. 260-293.

TeSNiERE, L., 1988, Eléments de syntaxe structurale, $2^{\mathrm{e}}$ éd. revue et corrigée (1 éd. 1959), Paris, Klincksieck.

TraugotT, E. C., 2002, Regularity in semantic change. Cambridge, Cambridge University Press.

\section{Euvres et auteurs cités Français médiéval}

Chretien de Troyes, Chevalier de la Charrette ou Lancelot, P. Kunstmann (éd.), Ottawa, Nancy, Université d'Ottawa, Laboratoire de Français Ancien, ATiLF, 2009. Publié en ligne par la BFM.

Queste del saint Graal, C. Marchello-Nizia et A. Lavrentiev (éds.), Lyon, Equipe BFM, 2013. Publié en ligne par la BFM.

RaOul De Houdenc, Meraugis de Portlesguez, M. Szkilnik (éd.), Genève, Honoré Champion, 2004.

Renaut de Beaujeu, Bel Inconnu, P. Williams (éd.), Paris, Champion, 1929. Publié en ligne par la BFM.

Tristan en prose, P. Ménard (éd.), Genève, Librairie Droz, 1987.

Français préclassique et classique (textes publiés en ligne par Frantext)

BeAUMARCHAIS, Le Mariage de Figaro, Paris, Gallimard, 1988.

Bougeant, H., Voyage merveilleux du prince Fan-Férédin dans la Romancie, Paris, Le Mercier, 1735. 
GARNIER, R., Bradamante, Paris, Les Belles lettres, 1949.

— Les Juifves, Paris, Les Belles lettres, 1949.

GREVIN, J., Les Esbahis, Paris, Champion, 1980.

LA Fontaine, J. de, Fables : livres 9, 10, 11, Paris, Gallimard, 1991.

Lesage, A.-R., Achmet et Almanzine, Paris, Vve Pissot, 1728.

$\mathrm{M}^{\mathrm{ME}}$ DE SEVIGNE, Correspondance : tome 1, (1646-1675), Paris, Gallimard, 1972.

MARIVAUX, Le Petit-maître corrigé, Genève, Librairie Droz.

- Arlequin poli par l'amour, Paris, Garnier, 1968.

—L'épreuve, Paris, Garnier, 1968.

— La Vie de Marianne, partie 3, Paris, Gallimard, 1997.

MASURES, L. de, David fugitif, Paris, E. Cornély, 1907.

MOLIERE, Monsieur de Pourceaugnac, Paris, Hachette, 1882.

PuRE AbBe, M. de, La Prétieuse ou le Mystère des ruelles : Parties 1-2, Paris, Librairie Droz, 1938.

Quinault, P., Roland, Paris, C. Ballard, 1685.

SEDAINE, M.-J., Le Déserteur, Paris, Hachette, 1860. 